CYBERNETICS AND INFORMATION TECHNOLOGIES • Volume 15, No 7

Special Issue on Information Fusion

Sofia $\bullet 2015$

\title{
Selected Applications of Scale Spaces in Microscopic Image Analysis
}

\section{Dimiter Prodanov, Tomasz Konopczynski, Maciej Trojnar}

EHS, Interuniversity Microelectronics Center (IMEC), 3001 Leuven, Belgium

Emails:dimiter.prodanov@imec.be_dimiterpp@gmail.com

\begin{abstract}
Image segmentation methods can be classified broadly into two classes: intensity-based and geometry-based. Edge detection is the base of many geometrybased segmentation approaches. Scale space theory represents a systematic treatment of the issues of spatially uncorrelated noise with its main application being the detection of edges, using multiple resolution scales, which can be used for subsequent segmentation, classification or encoding. The present paper will give an overview of some recent applications of scale spaces into problems of microscopic image analysis. Particular overviews will be given to Gaussian and alpha-scale spaces. Some applications in the analysis of biomedical images will be presented. The implementation of filters will be demonstrated.
\end{abstract}

Keywords: Edge-detection, Gaussian filtering, alpha-stable distributions.

\section{Introduction}

Image segmentation is a non-trivial problem. There are several classes of methods, which can be applied in different circumstances. They can be classified broadly into two classes: intensity-based, where the hypothesis is that only the difference in the image histogram could be sufficient for segmentation; and geometry-based, where the image is transformed, so that the geometric features of interest become enhanced. There are various geometry-based segmentation approaches, for example, using 
edges, distances or texture statistics. In addition, there is a vast array of pre- and postprocessing techniques, such as smoothing, mathematical morphology operations (i.e., watershed), partial differential equation methods and shape methods. The present paper will focus on the geometry-based methods with a particular emphasis on the edge-detection techniques. Geometry-based approaches are invariant to changes in the illumination, which is an issue in natural images and some microscopic techniques. In contrast, geometry-based approaches are susceptible to structural or texture "noise", so that extra care must be taken to address such issues.

The edges are invariant geometric image features which can be used in various segmentation, classification or encoding algorithms. Most of the approaches for edge detection can be grouped into two categories, search-based and zero-crossing based. The search-based methods detect edges by first computing a measure of the edge strength, for example the gradient magnitude, and then searching for the local directional maxima of the gradient magnitude, using a computed estimate of the local orientation of the edge, usually the gradient direction. The zero-crossing based method estimates zeroes in second-order derivatives.

\section{Linear scale spaces}

Scale space theory represents a systematic treatment of the issues of spatially uncorrelated noise. It is well known that differentiation is an ill-posed problem and calculation of finite differences quotients is numerically unstable and amplifies the high frequency noise.

Scale spaces are derived from the physical principles of scale invariance. Since the images are finite physical signals, they are naturally related to the properties of the measurement apparatus. As idealization, these properties are described by a linear transfer function, so that the measurement process becomes a convolution. On the other hand, the measurement is always contaminated by an unwanted signal, which is denoted broadly as "noise". The noise process corresponds to non-linearities of the measurement step and the studied physical process. In many occasions, because of its irregularity in time, it can be treated as a purely random process. Since the physical measurement is a repeated process, the Gaussian noise comes as the simplest model by virtue of the Central Limit Theorem. Therefore, formally this reasoning can be described as

$$
I(t)=T(t) * f(t)+\Delta W_{t},
$$

where "*" denotes the convolution operation and the second term is the noise increment, which has "bad" (i.e., singular) behaviour and must be regularized. Since all digital samplings are discrete, they are also facing the additional challenge of estimating the continuous physical process from its samples.

\subsection{Gaussian scale spaces}

Gaussian kernels enjoy the property of separability. Therefore, the computation of convolutions can be simplified, if one-dimensional kernels are applied directionally in a sequence. In one spatial dimension the kernel can be expressed as 


$$
G_{s}(x)=\frac{1}{\sqrt{2 \pi s}} e^{-x^{2} / 2 s} .
$$

The shape of the increasing kernels is governed by the scale parameter $s$. The interactions of the Gaussian kernels with the image can be viewed as realizations of a diffusion process and can be described well by the diffusion equation:

$$
\frac{\partial L}{\partial s}=\frac{1}{2} \Delta L
$$

with an initial condition of the original image $L(x, y, 0)=I(x, y)$. This observation can be generalized by the concept of parametric scale spaces, generated by successive smoothing of the image with a Gaussian kernel. The special properties of the Gaussian kernel rely on the fact that it is a generic solution (i.e., a Green function) of the former diffusion equation. The diffusion in the image plane can therefore be expressed as a spatial convolution:

$$
L(x, y, s)=G_{s}(x, y) * I(x, y) .
$$

In the context of image processing, these properties have been used to construct the mathematical apparatus of scale space theory independently by Ijima [3] and his disciples in Japan, and Witkin [9] in Europe. The mathematical theory of the scale spaces is described in [8].

\subsection{Fractional scale spaces}

Pauwels et al. [7], and later Duits et al. [1] have investigated the use of the fractional powers of Laplacian operator in connection with the scale invariant smoothing and scale space theory. The approach leads to formulation and solving of a fractional heat problem:

$$
\frac{\partial L}{\partial s}=-\frac{1}{2}(-\Delta)^{\frac{\alpha}{2}} L, 0<\alpha \leq 2 .
$$

It can be shown that the fractional heat kernel arises in problems with $\alpha$-stable Levy noise. In the time/space domain the solution is given by a Levy $\alpha$-stable density $[2,4]$ :

$$
G_{S}(x)=\frac{1}{(s / 2)^{2 / \alpha}} S^{1-\alpha}\left(\frac{x}{(s / 2)^{1 / \alpha}}\right) S^{1-\alpha}\left(\frac{y}{(s / 2)^{1 / \alpha}}\right), \quad \alpha \leq 1,
$$

where the function $S^{1-\alpha}(x)$ is given by the infinite series:

$$
S^{1-\alpha}(x)= \begin{cases}\frac{1}{\pi} \sum_{n=1}^{\infty}(-1)^{n+1} \frac{\Gamma(1+n \alpha)}{x^{\alpha n+1} n !} \sin n \alpha \pi, & x>0, \\ 0, & 0 \leq x,\end{cases}
$$

and 


$$
G_{s}(x)=\frac{1}{(s / 2)^{2 / \alpha}} S^{2-\alpha}\left(\frac{x}{(s / 2)^{1 / \alpha}}\right) S^{2-\alpha}\left(\frac{y}{(s / 2)^{1 / \alpha}}\right), 1 \leq \alpha \leq 2,
$$

where the function $S^{2-\alpha}(x)$ is given by the infinite series:

$$
S^{2-\alpha}(x)= \begin{cases}\frac{1}{\pi} \sum_{n=1}^{\infty}(-1)^{n+1}|x|^{n-1} \frac{\Gamma\left(1+\frac{n}{\alpha}\right)}{n !} \sin \frac{n \pi}{\alpha}, & x \neq 0, \\ \frac{1}{\pi \alpha} \Gamma\left(\frac{1}{\alpha}\right) \sin \frac{\pi}{\alpha}, & 0=x,\end{cases}
$$

where $\Gamma(x)$ denotes Euler's Gamma function.

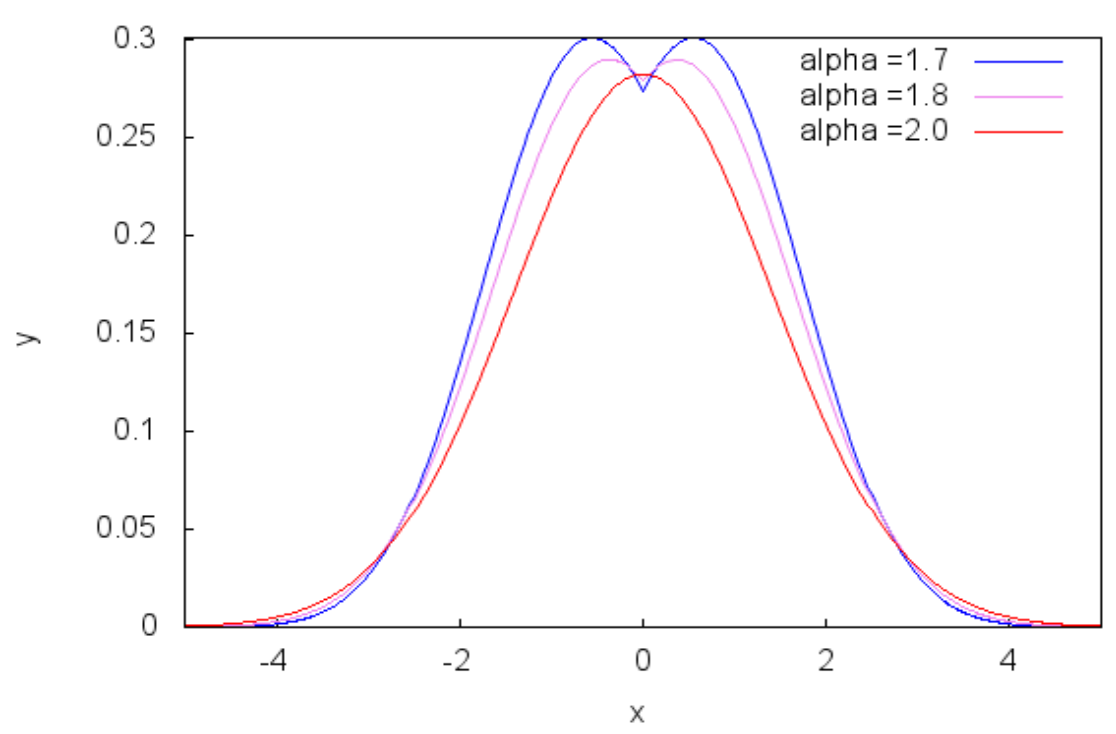

Fig. 1. Levy $\alpha$-stable distributions for three different values of the fractional order

The symmetrical stable density can be expressed in elementary or transcendent functions only for some choices of $\alpha$ exponents. Such cases are: $\alpha=2$, leading to Gaussian distribution; $\alpha=1$, leading to Cauchy distribution; and $\alpha=\frac{1}{2}$, leading to Levy-Smirnow distribution. These functions, on the other hand, can be conveniently represented in the Fourier domain as:

$$
\log S(\alpha, x)= \begin{cases}-\sigma^{\alpha}|x|^{\alpha}\left(1+i \beta \operatorname{sign}(x) \tan \pi \alpha / 2\left((\sigma|x|)^{1-\alpha}-1\right)\right)+i \mu x, & \alpha \neq 1, \\ \frac{2}{\pi} \sigma|x|(1+i \beta \operatorname{sign}(t) 2 / \pi \log (\sigma|t|))+i \mu x, & \alpha=1,\end{cases}
$$

in the asymmetrical case or

$$
\log S(\alpha, x)= \begin{cases}-\sigma^{\alpha}|x|^{\alpha}+i \mu x, & \alpha \neq 1 \\ \frac{2}{\pi} \sigma|x|+i \mu x, & \alpha=1\end{cases}
$$


for the completely symmetrical case. Note that the first formula corresponds to the latter case. This parametrization is a variant of Zolotariov's parametrization, jointly continuous in all parameters.

\subsection{Powers of Laplacian (PoL) operators}

Detection of the image features, such as extrema and edges, which depend of derivatives operations, is stabilized by pre-smoothing. Replacing the ordinary spatial derivatives by derivatives of blurred images has a strong regularizing effect in the sense of non-enhancement of the local extrema, when successive filters are applied, i.e., the image landscape becomes more and more flattened. In the English literature this approach was pioneered by [6], who introduced a pre-processing Gaussian convolution step. On theoretical grounds Iijima demonstrated that this makes a robust step in differentiation. Differentiation can be combined with smoothing in one step. The resulting kernel is also known as Mexican hat filter or Laplacian of Gaussian (LoG). The approach is mathematically equivalent to multiscale wavelet techniques, since LoG fulfils the wavelet admissibility condition [5]. Different powers of the Laplacian operators have an especially simple representation in the Fourier domain. It can be derived, based on the Gaussian derivative in the Fourier domain and the binomial theorem.

The Fourier transform of the Gaussian derivatives is given by

$$
\widetilde{G_{n}}(f)=(2 \pi i f s)^{n} e^{-2 \pi^{2} f^{2} s},
$$

where the order $n$ is integer.

\section{Integer-order powers:}

The power of the Laplacian operator can be expressed therefore as

$$
\widetilde{L_{n}}(k)=(-2)^{n}(\pi k s)^{2 n} e^{-2 \pi^{2} k^{2} s}
$$

for a radial wave number $k=\sqrt{\omega^{2}+\eta^{2}}$.

\section{Fractional-order powers:}

In the Fourier domain, the fractional heat kernel is expressed as

$$
\widetilde{G_{n}}(\omega, \eta, s)=e^{-\frac{s\left(|2 \pi \omega|^{\alpha}+|2 \pi \eta|^{\alpha}\right)}{2}} .
$$

Therefore, in the fractional domain the operator will be expressed as direct generalization:

$$
\widetilde{L_{n, \alpha}}(k)=(-2)^{n}(\pi k s)^{2 n} e^{-2^{\alpha-1} \pi^{\alpha}|k|^{\alpha} s} .
$$

Therefore, the kernel bandwidth can be controlled by the fractional power $\alpha$.

\section{Anisotropic decomposition of the Laplacian operator}

In another line of development, the LoG operator can be decomposed into orthogonal and tangential components [4]: 
where the components are given by

$$
\Delta G=\Delta_{\|} G+\Delta_{\perp} G,
$$

$$
\Delta_{\perp} G=\frac{1}{r_{x}^{2}+r_{y}^{2}} \cdot\left(r_{x}^{2} \nabla_{x x} G+2 r_{x} r_{y} \nabla_{x y} G+r_{y}^{2} \nabla_{y y} G\right),
$$

and

$$
\Delta_{\|} G=\frac{1}{r_{x}^{2}+r_{y}^{2}} \cdot\left(r_{x}^{2} \nabla_{x x} L-2 r_{x} r_{y} \nabla_{x y} G+r_{y}^{2} \nabla_{y y} G\right) .
$$

The weighting of the components is according to the direction of the gradient $\left(r_{x}=\nabla_{x} G, r_{y}=\nabla_{y} G\right)$. This operator is introduced in order to differentialy respond to blob-like or tubular structures. A result, demonstrating the properties of the operator is presented in Fig. 2C.

(A)

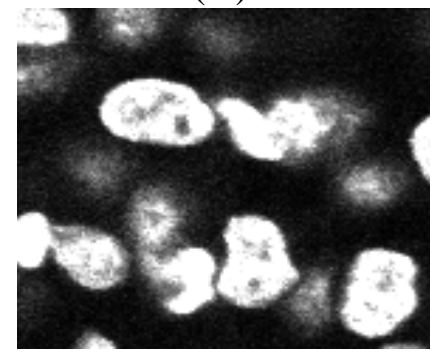

(B)

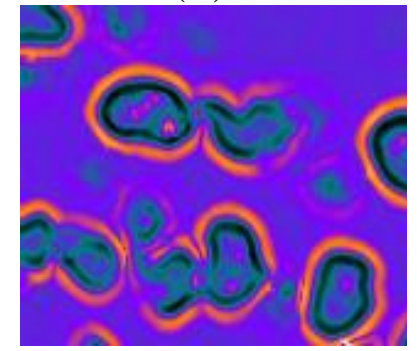

(C)

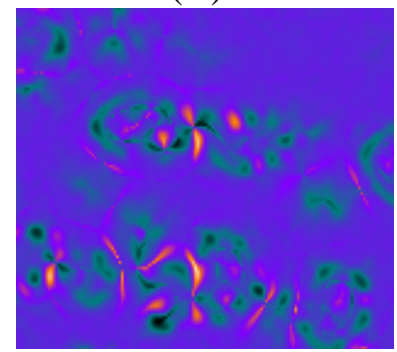

Fig. 2. LoG Anisotropic decomposition of a fluorescent microscopic image: Fluorescent microscopic image of cells (A); orthogonal LoG components (B); tangential LoG component (C), scale $\sigma=8.0$.

The intensity is represented as a heat map. Note the enhancement of the round shapes in the orthogonal component

The enhancement of the blob-like structures in an image can be used to improve the segmentation and allows the extraction of cell nuclei with inhomogeneous coloration distribution (Fig. 3C), which is not achievable using only histogram-based segmentation techniques.

(A)

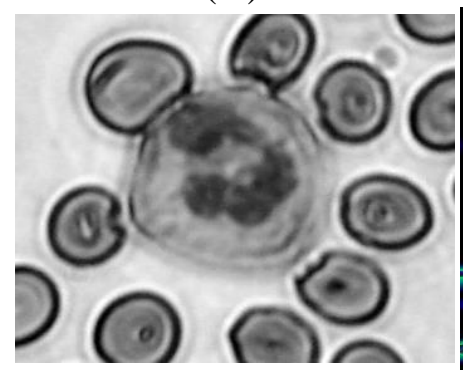

(B)

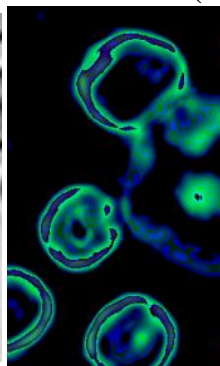

B)

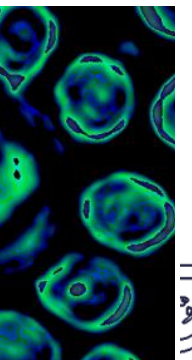

(C)

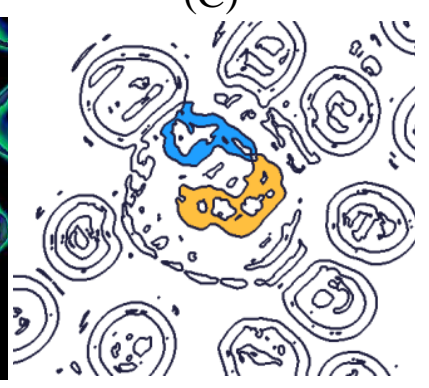

Fig. 3. Application of LoG anisotropic decomposition: Light microscopic image of a Giemsa-stained neutrophil cell touching on erythrocytes (A); median projection of LoG scale space across scales $\sigma=3.50-16.17$ (B); zero-crossings of the orthogonal component of LoG at $\sigma=6.83$ (C). Note the segmentation of the nuclear contour indicated by the color of the ROIs 


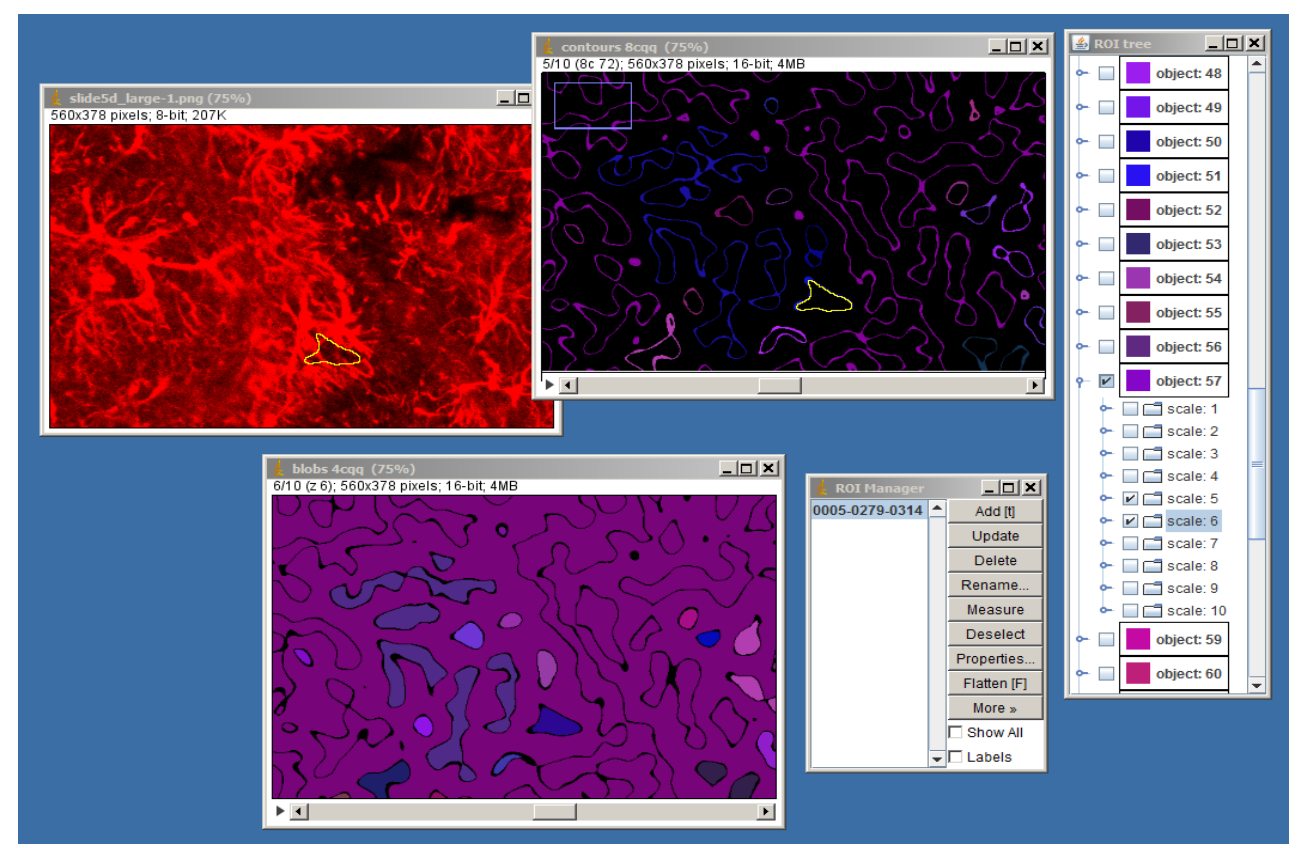

Fig. 4. Scale space ROI manager plugin

\section{Scale space regions of interest}

Gaussian-based filters are implemented in the public domain image analysis software ImageJ Zero-crossings of the Laplacean operator and its anisotropic decomposition can be assembled into $2 \mathrm{D}$ and $3 \mathrm{D}$ Regions Of Interest (ROIs) (Figs 2-4).

\section{Implementation}

The set of filters described in the present paper is implemented in ImageJ as a set of plugins. ImageJ is a public domain image processing program, written in Java. Since its inception in 1997, ImageJ has evolved to become a standard analytical tool in life sciences. It has an open architecture, providing extensibility via 3rd party Java modules (called plugins) and scripting macros. It is developed by Wayne Raspband in 1997, and expanded via a contributed software code by an international group of contributors. Plugins are distributed together with their source code under various licences determined by the plugin authors.

We have used two implementation strategies: the integer order fliters are implemented in the spatial domain, while the fractional order filters are implemented in the Fourrier domain.

Acknowledgments: The work has been supported in part by Grants from the Research Fund - Flanders (FWO), contract No 0880.212.840 and ERC Consolidator Grant "SCALPEL". 


\section{References}

1. D u it s, R., M. F e $1 \mathrm{~s}$ b e r g, L. F 1 o r a c k, B. P 1 a t e 1. Alpha-Scale Spaces on a Bounded Domain. - Scale Space Methods in Computer Vision. Springer, 2003, pp. 494-510.

2. Hu a n g, F., F. Li u. The Space-Time Fractional Diffusion Equation with Caputo Derivatives. J. Appl. Math. and Computing, Vol 1-2, 2005, pp. 179-190.

3. I i j i m a, T. Theory of Pattern Recognition. - Electronics and Communications in Japan, Tokyo, 1963, pp. 123-134.

4. Ma in ardi, F., Y. Luchko, G. Pagnini. The Fundamental Solution of the Space-Time Fractional Diusion Equation. - Fract. Calc. Appl. Anal., Vol. 4, 2001, No 2, pp. 153-192.

5. Mallat, S., S. Zhong. Characterization of Signals from Multiscale Edges. - Vol. 14, 1992, No 7, pp. 710-732.

6. M a r r, D., E. H i ld r e th. Theory of Edge Detection. - In: Proc. R. Soc. Lond. B, Vol. 207, 1980, pp. 187-217.

7. P a u w e $1 \mathrm{~s}$, E. J., L. J. V an G o o 1, P. Fi d d e 1 a e r s, T. M o on s. An Extended Class of ScaleInvariant and Recursive Scale Space Filters. - IEEE Trans. Pattern Analysis and Machine Intel., Vol 17, 1995, No 7, pp. 691-701.

8. W e i c k e r t, J. Anisotropic Diffusion in Image Processing. ECMI Series. Stuttgart, TeubnerVerlag, 1998.

9. W i t k i n, A. P. Scale-Space Filtering. - In: Proc. of 8th Int. Joint Conf. Artificial Intelligence (IJCAI'83), Vol. 2, 8-12 August 1983, pp. 1019-1022. 